Pacific Journal of Mathematic 


\title{
ON SINGULAR INDICES OF ROTATION FREE DENSITIES
}

\author{
HIDEO IMAI
}

The properties of singular indices of nonnegative rotation free densities on $\Omega(\lambda)=\{\lambda<|z| \leqq 1\}(\lambda \geqq 0)$ will be studied. The relation between the singular index $\alpha(P)$ of a nonnegative rotation free density $P$ on $\Omega(\lambda)(\lambda>0)$ and the Martin compactification $\Omega(\lambda)_{P}^{*}$ of $\Omega(\lambda)$ with respect to the elliptic equation $\Delta u=P u$ will be established.

A nonnegative locally Hölder continuous function $P(|z|)(|z|=r)$ on $\Omega(\lambda)$ is called a nonnegative rotation free density on $\Omega(\lambda)$. The singular index $\alpha(P)$ of a density $P$ at $r=\lambda$ is the quantity given by

$$
\alpha(P)=\lim _{r \rightarrow \lambda} e_{1}(r) / e_{0}(r),
$$

where $e_{j}(r)(j=1,2)$ is a unique bounded solution of the equation

$$
\frac{d^{2}}{d r^{2}} e(r)+\frac{1}{r} \frac{d}{d r} e(r)-\left(P(r)+j^{2} / r^{2}\right) e(r)=0 \quad(j=0,1)
$$

on $(\lambda, 1]$ with $e_{j}(1)=1$ for $\lambda=0$, and furthermore with $\lim _{r \rightarrow \lambda} e_{j}(r)=$ 0 for $\lambda>0$. In particular, $\alpha(0)$ with $P \equiv 0$ on $\Omega(\lambda)(\lambda \geqq 0)$ will be referred to as the harmonic index at $r=\lambda$. The elliptic dimension, including the harmonic dimension with $P \equiv 0$, of a density $P$ at the ideal boundary $|z|=\lambda, \operatorname{dim} \mathscr{P}(\lambda)$ in notation, is the dimension of the half module $\mathscr{P}(\lambda)$ of the positive solutions $u$ of $\Delta u=P u$ on $\Omega(\lambda)$ with $u=0$ on $|z|=1$. The elliptic dimension is an ideal boundary property (M. Heins [2], K. Hayashi [1], and M. Ozawa [12], [13]). The Picard principle is said to be valid for a density $P$ at $|z|=\lambda$ if $\operatorname{dim} \mathscr{P}(\lambda)=1$ or equivalently $\alpha(P)=0$ (M. Nakai $[8])$.

In [8] M. Nakai had shown that the singular index $\alpha(P)$ at $\lambda=0$ determines the Martin compactification in the following ways:

$$
\Omega(0)_{P}^{*} \approx\{\alpha(P) \leqq|z| \leqq 1\}, \quad \alpha(P)=0 \text { or } \alpha(P)>0
$$

in the sense of homeomorphism for any nonnegative rotation free density $P$ on $\Omega(0)$ and each ideal boundary point is minimal.

It will be shown that the singular indices are linearly ordered in the sense that $\alpha(P) \leqq \alpha\left(P_{1}\right)$ if $P \leqq P_{1}$ on $\Omega(\lambda)(\lambda \geqq 0)$. In particular, the harmonic index which is equal to $(-2 \lambda \log \lambda)\left(1-\lambda^{2}\right)^{-1}$ for $\lambda>0$ minimizes the singular indices at $r=\lambda$ among the nonnegative rotation free densities on $\Omega(\lambda)$. As a counterpart of 
the result of $M$. Nakai listed above, we will have that

$$
\Omega(\lambda)_{P}^{*} \approx\{\alpha(P) \leqq|z| \leqq 1\} \quad(\lambda>0)
$$

and each boundary point is minimal for any rotation free density $P \geqq 0$ on $\Omega(\lambda)$. Thus, the Picard principle is not valid on a hyperbolic end $\lambda<|z|<1(\lambda>0)$ for any rotation free density $P(r) \geqq 0$ on $\Omega(\lambda)$.

From the other aspect, a net $\{\Omega(\lambda)\}_{(\lambda>0)}$ may be viewed as a regular exhaustion of a parabolic end $\Omega(0)$ (precisely, $0<|z|<1$ ). Consider a density $P(r) \geqq 0$ on $\Omega(0)$. Then, $\alpha_{\lambda}(P)$ at $r=\lambda>0$ can be defined regarding $P(r)$ as a density on $\Omega(\lambda)$ in the same way as $\alpha(P)$ at $\lambda=0$. It will be shown that $\alpha_{\lambda}(P)$ is a decreasing function of $\lambda$ on $[0,1)$ and that

$$
\alpha(P)=\lim _{\lambda \rightarrow 0} \alpha_{\lambda}(P) .
$$

Also, the interrelation of generators of $\mathscr{P}(\lambda)$ and $\mathscr{P}(0)$ will be studied.

As for the Martin compactification with respect to the elliptic operator, we can find the work of S. Ito [4] and L. Hunt-R. Wheeden [3], among others. Neither the regularity of an elliptic operator nor the continuity of the solutions are assumed at the ideal boundary in the recent research on the Picard principle for which we refer to M. Nakai [8], [10], and [11], among others.

Finally, the author wishes to express his indebtness to Professor M. Nakai for his valued advice. Also, the discussion with the author's colleague Professor Shigeo Segawa is very helpful to the author in the preparation of this note.

1. Let $P(r)$ be any rotation free density on $\lambda<|z| \leqq 1(\lambda \geqq 0)$ and consider the elliptic differential equation

$$
L_{n} e(\boldsymbol{z}) \equiv L_{P, n} e(\boldsymbol{z})=\Delta e(\boldsymbol{z})-\left(P(|z|)+n^{2} /|\boldsymbol{z}|^{2}\right) e(\boldsymbol{z})=0(n=0,1, \cdots)
$$

on $\lambda<|z| \leqq \rho$ for any $\rho$ fixed in $(\lambda, 1]$. We will denote by $f(r: \rho)$ a function defined on $\lambda<|z| \leqq \rho$ and by $f(r)$ the function $f(r: 1)$.

Let $e_{n}(r: \rho)(n \geqq 0)$ be the rotation free solution of $L_{n} e(z)=0$ on $\lambda<|z| \leqq \rho(|z|=r)$ with $\lim _{r \rightarrow \lambda} e_{n}(r: \rho)=0$ and $e_{n}(\rho: \rho)=1$ for $\lambda>0$, and the unique bounded rotation free solution of $L_{n} e(z)=0$ with $e_{n}(\rho: \rho)=1$ for $\lambda=0$. The existence of such a solution can be deduced by the solvability of the Dirichlet problem, Harnack principle, and the maximum principle (cf [8]). However we note that the applications of the maximum principle for the operator $L_{n}$ can be made in quite different ways according as $\lambda>0$ and $\lambda=0$.

Consider another rotation free density $P_{1}(r)$ such that 


$$
P(r) \leqq P_{1}(r)
$$

on $\lambda<|z| \leqq 1$ and denote by $\bar{e}_{n}(r: \rho)$ the corresponding solution to the density $P_{1}(r)$, i.e., the solution of $L_{n} e(z)=L_{P_{1}, n} e(z)=0$ with the same properties as $e_{n}(r: \rho)$. Then,

$$
e_{n}(r: \rho)=e_{n}(r) / e_{n}(\rho), \quad \bar{e}_{n}(r: \rho)=\bar{e}_{n}(r) / \bar{e}_{n}(\rho)
$$

on $\lambda<|z| \leqq \rho$. The maximum principle (cf. e.g., [7]) or equivalently the comparison principle [9] yields that

$$
e_{0}(r: \rho) \geqq e_{1}(r: \rho) \geqq \cdots \geqq e_{n}(r: \rho) \geqq \cdots \geqq 0,
$$

and

$$
\begin{gathered}
\bar{e}_{0}(r: \rho) \geqq \bar{e}_{1}(r: \rho) \geqq \cdots \geqq \bar{e}_{n}(r: \rho) \geqq \cdots \geqq 0, \\
e_{n}(r: \rho) \geqq \bar{e}_{n}(r: \rho) \quad(n \geqq 0)
\end{gathered}
$$

on $\lambda<|z| \leqq \rho$. Setting

$$
v_{n}(r: \rho)=e_{n}(r: \rho) / e_{0}(r: \rho) \quad(n \geqq 1),
$$

we obtain from (1) and (2) that $v_{n}(r: \rho)=\left(e_{n}(r) / e_{0}(r)\right) /\left(e_{n}(\rho) / e_{0}(\rho)\right) \leqq 1$ for any $r$ and $\rho$ with $\lambda<r \leqq \rho \leqq 1$. Hence $v_{n}(r)$ is an increasing function of $r$ on $(\lambda, 1]$ and the same is true for $v_{n}(r: \rho)$ on $(\lambda, \rho]$, since $v_{n}(r: \rho)=v_{n}(r) / v_{n}(\rho)$ on $(\lambda, \rho]$. Thus we have

$$
\frac{d}{d r} v_{n}(r: \rho) \geqq 0 \quad(n \geqq 1)
$$

on $(\lambda, \rho]$ for any $\rho$ in $(\lambda, 1]$.

2. Consider the function $u_{n}(r)(n \geqq 0)$ on $(\lambda, 1]$ defined by

$$
u_{n}(r)=\bar{e}_{n}(r) / e_{n}(r) .
$$

Then, $u_{n}(r)(r=|z|)$ satisfies the elliptic equation

$$
\begin{gathered}
M_{n} u(z)=\Delta u(z)+2 \nabla \log e_{n}(|z|) \cdot \nabla u(z)-\left(P_{1}(|z|)-P(|z|)\right) u(z)=0 \\
(n \geqq 0)
\end{gathered}
$$

on $\lambda<|z|<1$, where $\nabla u$ is the gradient vector field $(\partial u / \partial x, \partial u / \partial y)$ of $u$. Since $\left(\bar{e}_{n}(r) / \bar{e}_{n}(\rho)\right) \leqq\left(e_{n}(r) / e_{n}(\rho)\right) \leqq 1$ on $(r, \rho]$ by (1) and (3), $u_{n}(r) \leqq u_{n}(\rho)$ on $(\lambda, 1]$ for any $r$ and $\rho$ in $(\lambda, 1]$ with $r<\rho$ which implies that

$$
\frac{d}{d r} u_{n}(r) \geqq 0 \quad(n \geqq 0)
$$

on $(\lambda, 1)$. Consequently, $\lim _{r \rightarrow \lambda} u_{n}(r)$ exists and $1>\lim _{r \rightarrow \lambda} u_{n}(r) \geqq 0$, because the constant 1 is not a solution of $M_{n} u(z)=0$. 
We define the function $h_{n}(r: \rho)$ by

$$
h_{n}(r: \rho)=\log \left(1 / e_{n}(r: \rho)\right) \text {. }
$$

Then, a direct computation yields that

$$
\begin{aligned}
M_{n} h_{n}(r)= & -\left[\nabla \log e_{n}(r) \cdot \nabla \log e_{n}(r)+P(r)+(n / r)^{2}+\left(P_{1}(r)\right.\right. \\
& \left.-P(r)) h_{n}(r)\right] \leqq 0 \quad(r=|z|)
\end{aligned}
$$

on $\lambda<|z|<1$. Hence the operator $M_{n}$ is parabolic for an end $\lambda<|z|<1$ (cf. [5] and [14]).

To assert that $u_{n}(r) \geqq u_{0}(r)(n \geqq 1)$ on $(\lambda, 1]$, observe that

$$
M_{n} u_{0}(r)=2 \nabla \log v_{n}\left(r^{*}\right) \cdot \nabla u_{0}(r) \geqq 0 \quad(r=|z|)
$$

on $\lambda<|z|<1$. Then, $M_{n}\left(u_{n}(r)-u_{0}(r)+\varepsilon h_{n}(r)\right) \leqq 0$ on $\lambda<|z|<1$ for every $\varepsilon>0$. Since $\lim _{r \rightarrow \lambda}\left(u_{n}(r)-u_{0}(r)+\varepsilon h_{n}(r)\right) \geqq 0$ and $u_{n}(r)-$ $u_{0}(r)+\varepsilon h_{n}(r)=0$ at $r=1$, the maximum principle implies that $u_{n}(r)-u_{0}(r)+\varepsilon h_{n}(r) \geqq 0$ on $\lambda<|z|<1$ for every $\varepsilon>0$. Letting $\varepsilon \rightarrow 0$, we deduce that

$$
u_{n}(r) \geqq u_{0}(r) \quad(n \geqq 1)
$$

on $(\lambda, 1]$.

3. We now define the quantity

$$
\alpha_{n}^{\prime}(P)=\lim _{r \rightarrow \lambda} v_{n}(r)
$$

for any nonnegative rotation free density $P(r)$ on $\lambda<|z| \leqq 1(\lambda \geqq 0)$ which will be referred to as the $n$th singular index of $P$ at $r=\lambda$. The quantity $\alpha_{1}(P)$ is abbreviated as $\alpha(P)$ and simply called the singular index of $P$ at $r=\lambda$ ([18]). In particular $\alpha(0)$ with $P=0$ will be referred to as the harmonic index at $r=\lambda$.

First, in view of $(5)$, we have $\bar{e}_{n}(r) / \bar{e}_{0}(r) \geqq e_{n}(r) / e_{0}(r)$ on $(\lambda, 1]$ and hence $\alpha_{n}\left(P_{1}\right) \geqq \alpha_{n}(P)$. It is needless to say that $\alpha_{n}\left(P_{1}\right)(n \geqq 1)$ indicate the $n$th singular index of $P_{1}$ at $r=\lambda$. For $P=0$ and $\lambda>0$, observe that $e_{0}(r)=\log (\lambda / r) / \log \lambda$ and $e_{1}(r)=\left(r-\lambda^{2} r^{-1}\right)(1-$ $\left.\lambda^{2}\right)^{-1}$. Then, by the L'Hospital theorem, we deduce that $\alpha(0)=$ $(-2 \lambda \log \lambda)\left(1-\lambda^{2}\right)^{-1}$. In the case of $\lambda=0$, beside $P=0$ there are many densities $P(r) \geqq 0$ which satisfies $\alpha(P)=0$ ([8], also see (34) in [6]). We summarize the result in:

THEOREM 1. Let $P(r)$ and $P_{1}(r)$ be any rotation free densities which satisfy the condition $P(r) \leqq P_{1}\left(r^{\circ}\right)$ on $\lambda<|z| \leqq 1(\lambda \geqq 0)$. Then,

$$
0 \leqq \alpha_{n}(P) \leqq \alpha_{n}\left(P_{1}\right)<1 \quad(n \geqq 1) \text {. }
$$


In particular, the harmonic index $\alpha(0)$ at $r=\lambda$ which is equal to $(-2 \lambda \log \lambda)\left(1-\lambda^{2}\right)^{-1}$ for $\lambda>0$ minimizes singular indices among nonnegative rotation free densities on $\lambda<|z| \leqq 1$.

The above theorem intuitively indicates that in the case $\lambda>0$ we can make $\alpha(P)$ sufficiently close to 1 , selecting a sufficiently large density $P$. To see our inspection is certainly true, we first observe that

$$
L_{0}\left((r / \rho)^{m}-e_{0}(r: \rho)\right)=\left(r^{m-2} / \rho^{m}\right)\left(m^{2}-P(r) r^{2}\right)
$$

on $\lambda<|z| \leqq \rho$ with $r=|z|$. Then, for an $m>0$ satisfying

$$
m^{2}-P(r) r^{2} \leqq 0
$$

on $\lambda<|z| \leqq 1,(r / \rho)^{m} \geqq e_{0}(r: \rho)$ is valid on $(\lambda, \rho]$ by the maximum principle and hence we deduce that $d / d r\left(e_{0}(r) / r^{m}\right) \geqq 0$ on $(\lambda, 1]$ for such an $m>0$. Thus we can easily see that

$$
L_{1}\left(e_{1}(r)-r^{c} e_{0}(r)\right)=-2 c r^{c-1} e_{0}(r) \frac{d}{d r} \log \left(e_{0}(r) / r^{m}\right) \leqq 0
$$

on $(\lambda, 1)$ for any $c$ fixed in $(0,1]$ and satisfying (6) with $m=(1-$ $\left.c^{2}\right) / 2 c$. A fortiori, $e_{1}(r) / e_{0}(r) \geqq r^{c}$ on $(\lambda, 1]$. Thus we deduce:

Proposition 1. Let $P(r)$ be any rotation free density on $\lambda<$ $|z| \leqq 1(\lambda>0)$ which satisfies the condition (6) with $m=\left(1-c^{2}\right) / 2 c$, $c$ being fixed in $(0,1]$. Then,

$$
\lambda^{c} \leqq \alpha(P)<1
$$

is valid.

4. We observe that the function $v_{n}(r: \rho)(r=|z|)$ in no. 1 is a bounded solution of the elliptic equation

$$
I_{n} v(z)=\Delta v(z)+2 \nabla \log e_{0}(|z|: \rho) \cdot \nabla v(z)-(n / r)^{2} v(z)=0 \quad(n \geqq 1)
$$

on $\lambda<|z| \leqq \rho$. A direct computation yields that

$$
I_{n} h_{0}(r: \rho)=-\left[P(r)+\nabla \log e_{0}(r: \rho) \cdot \nabla \log e_{0}(r: \rho)+(n / r)^{2} h_{0}(r: \rho)\right]<0
$$

on $\lambda<|z|<\rho$. Hence each operator $I_{n}$ possesses an Evans supersolution $h_{0}(r: \rho)$ on $\lambda<|z|<\rho$ (cf. e.g., [5], [14]).

To compare $v_{n}(r: \rho)$ for $n=1,2, \cdots$, we observe that, for $m>0$,

$$
\begin{gathered}
I_{n}\left[v_{1}(r: \rho)^{m}\right]=m(m-1) v_{1}(r: \rho)^{m-2}\left(\frac{d}{d r} v_{1}(r: \rho)\right)^{2} \\
-\left(\left(n^{2}-m\right) / r^{2}\right) v_{1}(r: \rho)^{m} \quad(r=|z|)
\end{gathered}
$$


is valid on $\lambda<|z|<\rho$. Taking $m=n^{2}, \quad I_{n}\left[v_{1}(r: \rho)^{n^{2}}\right] \geqq 0$ on $\lambda<$ $|z|<\rho$ for $n>1$. Also, setting $m=n$,

$$
I_{n}\left[v_{1}(r: \rho)^{n}\right]=n(n-1) v_{1}(r: \rho)^{n-2}\left[\left(\frac{d}{d r} v_{1}(r: \rho)\right)^{2}-\left(v_{1}(r: \rho) / r\right)^{2}\right]
$$

on $\lambda<|z|<\rho$. Considering $L_{1}\left(e_{1}(r: \rho)-(r / \rho) e_{0}(r: \rho)+\varepsilon \log (r / \rho)\right)$ on $\lambda<|z|<\rho$ with every $\varepsilon>0$ for $\lambda=0$ and $\varepsilon=0$ for $\lambda>0$. Then, $v_{1}(r) / r \geqq v_{1}(\rho) / \rho$ is valid for any $r$ and $\rho$ such that $\lambda<r \leqq \rho \leqq 1$. This inequation shows that $v_{1}(r) / r$ is decreasing on $(\lambda, 1]$, and the same is true for $v_{1}(r: \rho) / r$ on $(\lambda, \rho]$. Hence, $d / d r \log \left(v_{1}(r: \rho) / r\right) \leqq 0$ on $(\lambda, \rho]$, and a fortiori $d / d r v_{1}(r: \rho)-v_{1}(r: \rho) / r \leqq 0$ on $(\lambda, \rho)$. Consequently, $I_{n}\left[v_{1}(r: \rho)^{n}\right] \leqq 0$ on $\lambda<|z|<\rho$. From these estimates it can be deduced that $I_{n}\left[v_{n}(r: \rho)-v_{1}(r: \rho)^{n^{2}}+\varepsilon h_{0}(r: \rho)\right] \leqq 0 \quad(n \geqq 2)$ and $I_{n}\left[v_{1}(r: \rho)^{n}-v_{n}(r: \rho)+\varepsilon h_{0}(r: \rho)\right] \leqq 0(n \geqq 1)$ on $\lambda<|z|<\rho$ for every $\varepsilon>0$. Therefore, the maximum principle yields that

$$
v_{1}(r: \rho)^{n^{2}} \leqq v_{n}(r: \rho) \leqq v_{1}(r: \rho)^{n} \quad(n \geqq 1)
$$

on $\lambda<|z| \leqq \rho$ for any $\rho$ in $(\lambda, 1]$. Taking $\rho=1$ in the above inequations, we can deduce:

LEMMA 1. For any rotation free density $P(r) \geqq 0$ on $\lambda<|z| \leqq$ $1(\lambda \geqq 0)$,

$$
\alpha(P)^{n^{2}} \leqq \alpha_{n}(P) \leqq \alpha(P)^{n} \quad(n \geqq 1)
$$

5. Consider the Green's function $G(z, \zeta)$ of $\Delta u=P u$ on $\lambda<|z|<$ $1(\lambda \geqq 0)$ with pole at $\zeta$. For any $z$ and $\zeta=\mathrm{re}^{i \sigma}$ in $\lambda<|z|<1$ with $|z| \neq r$, expand $G(z, \zeta)$ into Fourier series:

$$
G\left(z, \mathrm{re}^{i \sigma}\right)=c_{0}(z: r) / 2+\sum_{n=1}^{\infty}\left(c_{n}(z: r) \cos n \sigma+s_{n}(z: r) \sin n \sigma\right),
$$

where

$$
\begin{aligned}
c_{n}(z: r) & =\pi^{-1} \int_{0}^{2 \pi} G\left(z, \mathrm{re}^{i \sigma}\right) \cos n \sigma d \sigma \quad(n \geqq 0), \quad s_{n}(z: r) \\
& =\pi^{-1} \int_{0}^{2 \pi} G\left(z, \mathrm{re}^{i \sigma}\right) \sin n \sigma d \sigma \quad(n \geqq 1) .
\end{aligned}
$$

Since $G(z, \zeta)$ is dominated by the harmonic Green's function $\log (|1-\bar{\zeta} z| /|z-\zeta|)$ of the unit disk, the method of M. Nakai [8] can be modified to the hyperbolic case. But for the sake of completeless, we draw the outline of the method in [8]. The $c_{n}(z: r)$ $(n \geqq 0)$ and $s_{n}(z: r)(n \geqq 1)$ are bounded continuous functions of $r$ on $(\lambda, 1]$, and satisfy $L_{n} c_{n}(z: r)=0 \quad(n \geqq 0)$ and $L_{n} s_{n}(z: r)=0 \quad(n \geqq 1)$ for $r \neq|z|$. Therefore 


$$
c_{n}(z: r) / c_{n}(z: \rho)=e_{n}(r: \rho), \quad s_{n}(z: r) / s_{n}(z: \rho)=e_{n}(r: \rho)
$$

for every $r$ and $\rho$ with $\lambda<r \leqq \rho<|z|$, and

$$
\left|c_{n}(z: \rho)\right|,\left|s_{n}(z: \rho)\right| \leqq c_{0}(z: \rho) \leqq 2 \min \left(\log \rho^{-1}, \log |z|^{-1}\right) .
$$

Since $\sup _{r \in\langle\lambda, \tau]} v_{n}(r: \rho)=\rho_{\tau}<1$ for any $\tau$ such that $\tau<\rho$, by the Weierstrass double convergence theorem, the limit

$$
L(z, \theta: \rho)=\lim _{r \rightarrow \lambda, \sigma \rightarrow \theta} G\left(z, \mathrm{re}^{i \sigma}\right) / e_{0}(r: \rho)
$$

exists for any $\rho$ in $(\lambda, 1]$ with $\rho<|z|$ which is possitive function of $z$ on $\rho<|z|<1$, where

$$
\begin{aligned}
L(z, \theta: \rho)= & c_{0}(z: \rho) / 2+\sum_{n=1}^{\infty}\left(c_{n}(z: \rho) \cos n \theta\right. \\
& \left.+s_{n}(z: \rho) \sin n \theta\right) \alpha_{n}(P) v_{n}(\rho)^{-1}
\end{aligned}
$$

for any $z, \rho$ such that $\lambda<\rho<|z|<1$, and any $\theta$ in $T$. Denoting $G(z, \zeta) / G\left(z_{0}, \zeta\right)$ by $K(z, \zeta)$ for some fixed $z_{0}$ in $\lambda<|z|<1$, we obtain from the relation $K\left(z, \mathrm{re}^{i \sigma}\right)=\left(G\left(z, \mathrm{re}^{i \sigma}\right) / e_{0}(r: \rho)\right) /\left(G\left(z_{0}, \mathrm{re}^{i \sigma}\right) / e_{0}(r: \rho)\right)$ that

$$
\lim _{r \rightarrow \lambda, \sigma \rightarrow \theta} K\left(z, \mathrm{re}^{i \sigma}\right)=L(z, \theta: \rho) / L\left(z_{0}, \theta: \rho\right)
$$

for every $z$ in $\lambda<|z| \leqq 1$ and any $\theta$ in $T \equiv(-\infty, \infty) / \bmod 2 \pi$, where $\rho$ is any number in $(\lambda,|z|) \cap\left(\lambda,\left|z_{0}\right|\right)$. Since the left hand side of the above equation is independent of $\rho$, we can define the function

$$
k(z, \theta)=L(z, \theta: \rho) / L\left(z_{0}, \theta: \rho\right)
$$

on $\lambda<|z| \leqq 1$ for any $\theta$ in $T$ and any $z_{0}$ in $\lambda<|z|<1$.

In passing we note that the following result which had shown for $\lambda=0$ in [8] is also valid for $\lambda>0$, since the proof in [8] is not affected by the parabolicity of the origin.

LEMMA 2. The system $c_{n}(z: r) \quad(n \geqq 0)$ and $s_{n}(z: r) \quad(n \geqq 1)$ is linearly independent as functions of $z$ in the following sense: Let $\sum_{n=0}^{\infty} a_{n}$ and $\sum_{n=1}^{\infty} b_{n}$ be arbitrary absolutely convergent real series. Suppose that

$$
\sum_{n=0}^{\infty} a_{n} c_{n}(z: r)+\sum_{n=1}^{\infty} b_{n} s_{n}(z: r)=0
$$

on a nonempty open subset of $\lambda<|z|<1(\lambda>0)$ for any fixed $r$ in $(\lambda, 1)$. Then, $a_{0}=a_{n}=b_{n}=0(n \geqq 1)$.

6. We denote $\lambda<|z| \leqq 1$ by $\Omega(\lambda)$ and consider the Martin compactification $\Omega(\lambda)_{F}^{*}$ of an relatively closed annulus $\Omega(\lambda)(\lambda>0)$ 
with respect to $\Delta u=P u$ toward the ideal boundary $|z|=\lambda$. As usual, the Martin topology can be given by the completion of $\Omega(\lambda)$ with respect to the metric $d$ defined by

$$
d\left(\zeta_{1}, \zeta_{2}\right)=\sum_{n=1}^{\infty} \frac{1}{2^{n}} \frac{\left|K\left(z_{n}, \zeta_{1}\right)-K\left(z_{n}, \zeta_{2}\right)\right|}{1+\left|K\left(z_{n}, \zeta_{1}\right)-K\left(z_{n}, \zeta_{2}\right)\right|}
$$

for any $\zeta_{1}$ and $\zeta_{2}$ in $\Omega(\lambda)$, where $\left\{z_{n}\right\}_{n=1}^{\infty}$ is any dense subset of $\lambda<$ $|z|<1$. A point $\zeta^{*}$ in $\Gamma_{P}=\Omega(\lambda)_{P}^{*}-\Omega(\lambda)$ is called minimal if $K\left(z, \zeta^{*}\right)$ is minimal in the half module $\mathscr{P}(\lambda)$ of the positive solutions $u$ of $\Delta u=P u$ on $\Omega(\lambda)$ with $u=0$ on $|z|=1$. Since $\alpha(P)>0$, we can deduce by the same arguments as in [8] that each $k(z, \theta)$ is minimal, and that $k\left(z, \theta_{1}\right)$ and $k\left(z, \theta_{2}\right)$ are nonproportional for each pair $\theta_{1} \neq \theta_{2}$ in $T$ by virtue of Lemma 2. Then, the relation between $k(z, \theta)$ and $K\left(z, \zeta^{*}\right)$ are given by

$$
k(z, \theta)=K\left(z, \arg \pi\left(\zeta^{*}\right)\right)
$$

for any $\zeta^{*}$ in $\Gamma_{P}$ with $\theta=\arg \pi\left(\zeta^{*}\right)$, where $\pi$ is the homeomorphism of $\Omega(\lambda)_{P}^{*}$ onto the closed annulus $\lambda \leqq|z| \leqq 1$. Denoting by $(r, \theta)$ the polar coordinate of a point $\zeta$ in $\lambda \leqq|z| \leqq 1$, the mapping $\tau \cdot \pi$ defines a homeomorphism of $\Omega(\lambda)_{P}^{*}$ onto $\alpha(P) \leqq|z| \leqq 1$, where $\tau$ is given by $\tau(r, \theta)=(1-\lambda)^{-1}[(1-\alpha(P)) r+\alpha(P)-\lambda] e^{i \theta}$. Thus we have the counterpart of Theorem 5 in M. Nakai [8].

THEOREM 2. The Martin compactification $\Omega(\lambda)_{P}^{*}$ of $\Omega(\lambda)(\lambda>0)$ with respect to any rotation free density $P(r) \geqq 0$ on $\Omega(\lambda)$ is homeomorphic to the closed annulus $\alpha(P) \leqq|z| \leqq 1$ in such a way that every boundary point is minimal.

As an immediate consequence of Theorems 1 and 2, we have

$$
\operatorname{dim} \mathscr{P}(\lambda)=c \quad(\lambda>0),
$$

$c$ being the cardinal number of continuum. Thus, we conclude:

COROLlary. The Picard principle does not hold on a hyperbolic end $\Omega(\lambda)(\lambda>0)$ for any nonnegative rotation free density on $\Omega(\lambda)$.

In contrast to $\lambda>0, \operatorname{dim} \mathscr{P}(0)=1$ or $c$ for any nonnegative rotation free density $P$ on $0<|z| \leqq 1$ ([8]). Thus the comparison of the elliptic dimension is meaningful for $\lambda=0$. As a byproduct of Theorem 1, and Theorem 5 in [8], we have the following proposition which was shown as a consequence of the $b$-test by $M$. Nakai [9]. 
Proposition 2. Let $P$ and $P_{1}$ be any nonnegative rotation free densities such that $P(r) \leqq P_{1}(r)$ on $0<|z|=r \leqq 1$. Then,

$$
\operatorname{dim} \mathscr{P}(0) \leqq \operatorname{dim} \mathscr{P}_{1}(0),
$$

where $\operatorname{dim} \mathscr{P}_{1}(0)$ is the elliptic dimension of $P_{1}$ at $|z|=0$.

7. Consider a nonnegative rotation free density $P$ on $0<|z| \leqq$ 1 which will be fixed in the sequel. Then beside the $n$th singular index $\alpha_{n}(P)(n \geqq 1)$ of $P$ at $r=0$, we can define the $n$th singular index $\alpha_{n \lambda}(P)$ of $P$ at $r=\lambda$ for any $\lambda$ in $(0,1)$, regarding $P$ as a density on $\lambda<|z| \leqq 1$. We are concerned with an interrelation of the $\alpha_{n}(P)$ at $r=0$ and the $\alpha_{n \lambda}(P)$ at $r=\lambda$. To avoid the confusion in notations, we will denote any function $f_{n}(r: \rho)$ by $f_{n \lambda}(r: \rho)$ for $\lambda>0$ and $f_{n}(r: \rho)$ for $\lambda=0$ which are defined for a density $P$ on $0<|z| \leqq 1$. Likewise, $I_{n \lambda}$ stands for the operator $I_{n}$ in no. 4 considered on $\lambda<|z|<\rho$.

We consider the function $v_{n \lambda}(r: \rho)$ and $v_{n \eta}(r: \rho)$ for any $\lambda, \eta$, and $\rho$ with $0<\lambda \leqq \eta<\rho<1$. Since $I_{n \eta} v(z)=\Delta v(z)+2 \nabla \log e_{0 \eta}(|z|: \rho)$. $\nabla v(z)-(n /|z|)^{2} v(z)=0$ on $(\eta, \rho)$, we have by a direct computation

$$
\begin{aligned}
I_{n \eta} h_{0 \eta}(r: \rho)=-[P(r) & +\nabla \log e_{0 \eta}(r: \rho) \cdot \nabla \log e_{0 \eta}(r: \rho) \\
& \left.+(n / r)^{2} h_{0 \eta}(r: \rho)\right] \leqq 0
\end{aligned}
$$

and

$$
I_{n \eta} v_{n \lambda}(r: \rho)=2 \nabla \log \left(e_{0 \eta}(r: \rho) / e_{0 \lambda}(r: \rho)\right) \cdot \nabla v_{n \lambda}(r: \rho)
$$

on $\eta<|z|<\rho$. Then, since $1 \geqq e_{0 \eta}(r: \rho) / e_{0 \lambda}(r: \rho)=\left(e_{0 \eta}(r) / e_{0 \lambda}(r)\right) /\left(e_{0 \eta}(\rho) /\right.$ $\left.e_{0 \lambda}(\rho)\right)$ on $[\eta, \rho]$, the function $e_{0 \eta}(r) / e_{0 \lambda}(r)$ is increasing on $(\eta, 1)$ and the same is true for $e_{0 \eta}(r: \rho) / e_{02}(r: \rho)$ on $(\eta, \rho)$. Consequently $I_{n \eta} v_{n \lambda}(r: \rho) \leqq 0$ on $\eta<|z|<\rho$ and hence $I_{n \eta}\left(\varepsilon h_{0 \eta}(r: \rho)+v_{n \eta}(r: \rho)-\right.$ $\left.v_{n \lambda}(r: \rho)\right) \leqq 0$ on $\eta<|z|<\rho$ for every $\varepsilon>0$. Therefore it follows from the maximum principle that

$$
v_{n \lambda}(r: \rho) \leqq v_{n \eta}(r: \rho) \quad(n \geqq 1)
$$

on $(\eta, \rho)$ for any $\lambda, \eta$, and $\rho$ such that $0<\lambda \leqq \eta<\rho<1$. Since $v_{n \eta}(r: \rho)=v_{n \eta}(r) / v_{n \eta}(\rho), v_{n \lambda}(\eta) v_{n \lambda}(\rho)^{-1} \leqq \alpha_{n \eta}(P) v_{n \eta}(\rho)^{-1}$ and by the monotone increasingness of $v_{n \lambda}(r)$, we have

$$
\alpha_{n \lambda}(P) v_{n \lambda}(\rho)^{-1} \leqq \alpha_{n \eta}(P) v_{n \eta}(\rho)^{-1} \quad(n \geqq 1)
$$

for any $\lambda, \eta$, and $\rho$ with $0<\lambda \leqq \eta<\rho \leqq 1$.

Let $\bar{v}_{n \lambda}(r: \rho)$ be the continuous function on $[0,1]$ defined by $\bar{v}_{n \lambda}(r: \rho)=v_{n \lambda}(r: \rho)$ on $(\lambda, \rho]$ and $\bar{v}_{n \lambda}(r: \rho)=\alpha_{n \lambda}(P) v_{n \lambda}(\rho)^{-1}$ on $[0, \lambda]$ for any $\lambda$ in $(0,1)$. Since $\left\{\bar{v}_{n \lambda}(r: \rho)\right\}$ forms a decreasing net as $\lambda \rightarrow 0$, the limit $\bar{v}_{n}(r: \rho)=\lim _{\lambda \rightarrow 0} \bar{v}_{n \lambda}(r: \rho)$ exists. On the other hand, the 
convergence of $e_{n \lambda}(r: \rho) \rightarrow e_{n}(r: \rho)$ as $\lambda \rightarrow 0$ is uniform on $[\kappa, \rho]$ for any $\kappa$ in $(\lambda, \rho)$ by virtue of the Harnack principle. Thus $\left\{\bar{v}_{n \lambda}(r: \rho)\right\}$ converges uniformly to $v_{n}(r: \rho)$ on $[\kappa, \rho]$ and $\bar{v}_{n}(r: \rho)=v_{n}(r: \rho)$ on $[\kappa, \rho]$ for any $\kappa$ such that $0<\lambda<\kappa<\rho$. By the unique solvability of the Cauchy problem for $I_{n}$ on $0<|z|<\rho$ (cf. no. 4) we have $\bar{v}_{n}(r: \rho)=v_{n}(r: \rho)$ on $[0, \rho]$, setting $v_{n}(0, \rho)=\alpha_{n}(P) v_{n}(\rho)^{-1}$. Then $\lim _{\lambda \rightarrow 0} \bar{v}_{n \lambda}(r: \rho)=v_{n}(r: \rho)$, where $\left\{v_{n \lambda}(r: \rho)\right\}$ converges monotone decreasingly to the function $v_{n}(r: \rho)$ on $[0, \rho]$, and $\lim _{\lambda \rightarrow 0} \bar{v}_{n \lambda}(r: \rho)=$ $\alpha_{n}(P) v_{n}(\rho)^{-1}$ exists. Hence, by the Weierstrass double convergence theorem, we deduce that, for any $\lambda$ in $(0, \rho)$,

$$
\begin{aligned}
& \alpha_{n}(P) v_{n}(\rho)^{-1} \leqq \alpha_{n \lambda}(P) v_{n \lambda}(\rho)^{-1}, \\
& \quad \lim _{\lambda \rightarrow 0} \alpha_{n \lambda}(P) v_{n \lambda}(\rho)^{-1}=\alpha_{n}(P) v_{n}(\rho)^{-1} \quad(n \geqq 1) .
\end{aligned}
$$

Taking $\rho=1$ in the above discussion, we summarize the result in:

THEOREM 3. Let $P$ be any nonnegative rotation free density on $0<|z| \leqq 1$. Then, for any $\lambda$ and $\eta$ with $0<\lambda<\eta<1$,

$$
\alpha_{n \lambda}(P) \leqq \alpha_{n \eta}(P), \quad \lim _{\lambda \rightarrow 0} \alpha_{n \lambda}(P)=\alpha_{n}(P) \quad(n \geqq 1) .
$$

8. Consider a net $\{\lambda<|z|<1\}_{(\lambda>0)}$ which may be regarded as a regular exhaustion of a parabolic end $0<|z|<1$. The Green's functions of $\Delta u=P u$ on $\lambda<|z| \leqq 1$ and $0<|z| \leqq 1$ with pole at $\zeta$ are denoted by $G_{\lambda}(z, \zeta)$ and $G(z, \zeta)$, respectively. We maintain that for each $\theta$ fixed in $T$ a net of minimal functions $\left\{k_{\lambda}(z, \theta)\right\}$ converges to a minimal function $k(z, \theta)$ uniformly on each compact subset of $0<|z| \leqq 1$. We first note that in view of (8) the following inequalities are valid on $\eta \leqq|z| \leqq 1$ for any $\eta$ with $\rho<\eta$ : For $n=$ $1,2, \cdots$,

$$
\begin{aligned}
& c_{n}(z, \theta, \lambda) \equiv\left|c_{n \lambda}(z: \rho) \cos n \theta \alpha_{n \lambda}(P) v_{n \lambda}(\rho)^{-1}-c_{n}(z: \rho) \cos n \theta \alpha_{n}(P) v_{n}(\rho)^{-1}\right| \\
& s_{n}(z, \theta, \lambda) \equiv\left|s_{n \lambda}(z: \rho) \sin n \theta \alpha_{n \lambda}(P) v_{n \lambda}(\rho)^{-1}-s_{n}(z: \rho) \sin n \theta \alpha_{n}(P) v_{n}(\rho)^{-1}\right| \\
& \leqq \\
& \quad\left(2 \log \rho^{-1}\right)\left(\alpha_{n \lambda}(P) v_{n \lambda}(\rho)^{-1}-\alpha_{n}(P) v_{n}(\rho)^{-1}\right) \\
& \quad+\left\{\frac{1}{\pi} \int_{0}^{2 \pi}\left|G_{\lambda}\left(z, \rho e^{i \theta}\right)-G\left(z, \rho e^{i \theta}\right)\right| d \theta\right\} \alpha_{n}(P) v_{n}(\rho)^{-1}
\end{aligned}
$$

We have from (7) that $\alpha_{n \lambda}(P) v_{n \lambda}(\rho)^{-1} \leqq\left(\alpha_{1 \lambda}(P) v_{1 \lambda}(\rho)^{-1}\right)^{n}$ and $\alpha_{n}(P) v_{n}(\rho)^{-1}$ $\leqq\left(\alpha(P) v_{1}(\rho)^{-1}\right)^{n} \quad(n \geqq 1)$. Then by (8) and (9), $\left|L_{\lambda}(z, \theta: \rho)\right| \leqq$ $4\left(\log \eta^{-1}\right)\left(1-\alpha_{1 \lambda}(P) v_{1 \lambda}(\rho)^{-1}\right)^{-1}$ and $|L(z, \theta: \rho)| \leqq 4\left(\log \eta^{-1}\right)\left(1-\alpha(P) v_{1}(\rho)^{-1}\right)^{-1}$ on $\eta \leqq|z| \leqq 1$ which shows that both functions $L_{\lambda}(z, \theta: \rho)$ and $L(z$, $\theta: \rho)$ converge uniformly and absolutely on $\eta \leqq|z| \leqq 1$. Thus the function $\left|L_{\lambda}(z, \theta: \rho)-L(z, \theta: \rho)\right|$ is dominated by 


$$
\left|c_{0 \lambda}(z: \rho)-c_{0}(z: \rho)\right| / 2+\sum_{n=1}^{\infty}\left(c_{n}(z, \theta, \lambda)+s_{n}(z, \theta, \lambda)\right)
$$

on $\eta \leqq|z| \leqq 1$. Consequently, we have from the above estimates that

$$
\begin{aligned}
& \left|L_{\lambda}(z, \theta: \rho)-L(z, \theta: \rho)\right| \\
& \quad<\left\{\frac{2}{\pi} \int_{0}^{2 \pi}\left|G_{\lambda}\left(z, \rho e^{i \theta}\right)-G\left(z, \rho e^{i \theta}\right)\right| d \theta\right\}\left(1-\alpha(P) v_{1}(\rho)^{-1}\right)^{-1} \\
& \quad+\left(4 \log \rho^{-1}\right) \sum_{n=1}^{\infty}\left(\alpha_{n \lambda}(P) v_{n \lambda}(\rho)^{-1}-\alpha_{n}(P) v_{n}(\rho)^{-1}\right)
\end{aligned}
$$

on $\eta \leqq|z| \leqq 1$. Setting $\beta_{n}(\lambda)=\alpha_{n \lambda}(P) v_{n \lambda}(\rho)^{-1}-\alpha_{n}(P) v_{n}(\rho)^{-1}$,

and (14) yield that $\beta_{n}(\lambda)$ is a nonnegative function of $\lambda$ on $[0,1)$ which tends monotone decreasingly to 0 as $\lambda \rightarrow 0$. Also, $\sum_{n=1}^{\infty} \beta_{n}(\lambda)$ exists for each $\lambda$ in $[0,1)$, since $\sum_{n=1}^{\infty} \beta_{n}(\lambda) \leqq 2 \alpha_{n \lambda}(P) v_{n \lambda}(\rho)^{-1} /(1-$ $\left.\alpha_{n \lambda}(P) v_{n \lambda}(\rho)^{-1}\right)$. Thus we have $\lim _{\lambda \rightarrow 0} \sum_{n=1}^{\infty} \beta_{n}(\lambda)=0$. On the other hand, $\int_{0}^{2 \pi}\left|G_{\lambda}\left(z, \rho e^{i \theta}\right)-G\left(z, \rho e^{i \theta}\right)\right| d \theta$ tends to 0 uniformly on $\eta \leqq|z| \leqq 1$ as $\lambda \rightarrow 0$. Since $\eta$ is arbitrary in $(\rho, 1)$, we conclude that as $\lambda \rightarrow 0$ a net $\left\{L_{\lambda}(z, \theta: \rho)\right\}$ converges to the function $L(z, \theta: \rho)$ on each compact subset of $\rho<|z| \leqq 1$ for each $\theta$ fixed in $T$.

The functions $L_{\lambda}(z, \theta: \rho)$ and $L(z, \theta: \rho)$ are positive and finite on $\eta \leqq|z| \leqq 1$ for any $\eta$ in $(\rho, 1)$ (cf. no. 5). Taking $z_{0}$ in $\eta \leqq|z| \leqq 1$, the inequation

$$
\begin{aligned}
\mid k_{\lambda}(z, \theta)- & k(z, \theta)\left|\leqq L_{\lambda}\left(z_{0}, \theta: \rho\right)^{-1}\right| L_{\lambda}(z, \theta: \rho)-L(z, \theta: \rho) \mid \\
& +L_{\lambda}\left(z_{0}, \theta: \rho\right) L\left(z_{0}, \theta: \rho\right)^{-1} M\left|L\left(z_{0}, \theta: \rho\right)-L_{\lambda}\left(z_{0}, \theta: \rho\right)\right|
\end{aligned}
$$

is valid on $\eta \leqq|z| \leqq 1$, where $M=\max _{\eta \leqq|z| \leqq 1}|L(z, \theta: \rho)|$. As $\lambda \rightarrow 0$, $\left\{k_{k}(z, \theta)\right\}$ converges to $k(z, \theta)$ uniformly on $\eta \leqq|z| \leqq 1$. Since both functions $k_{\lambda}(z, \theta)$ and $k(z, \theta)$ can be defined independently of the choice of $\rho$ as far as $\rho<\min \left(\left|z_{0}\right|,|z|\right)($ cf. no. 5) and $\eta$ is arbitrary in $(\rho, 1)$, a net $\left\{k_{\lambda}(z, \theta)\right\}$ converges to the function $k(z, \theta)$ uniformly on each compact subset of $0<|z| \leqq 1$. Thus we have:

THEOREM 4. Let $P$ be any nonnegative rotation free density on $0<|z| \leqq 1$. Then, for each $\theta$ fixed in $T$, a net $\left\{k_{\lambda}(z, \theta)\right\}_{(\lambda>0)}$ converges to $k(z, \theta)$ uniformly on each compact subset of $0<|z| \leqq 1$ as $\lambda \rightarrow 0$, where $k_{\lambda}(z, \theta)$ and $k(z, \theta)$ are minimal functions with respect to $\Delta u=P u$ on $\lambda<|z| \leqq 1$ and $0<|z| \leqq 1$, respectively.

\section{REFERENCES}

1. M. Heins, Riemann surfaces of infinite genus, Ann. Math., 55 (1952), 296-317.

2. K. Hayashi, Les solutions positives de l'équation $\Delta u=P u$ sur une surface de Riemann, Kōdai Math. Sem. Rep., 13 (1961), 20-24. 
3. L. A. Hunt and R. L. Wheeden, Positive harmonic functions on Lipschitz domain, Trans. Amer. Math. Soc., 147 (1970), 507-527.

4. S. Ito, On the existence of Green function and positive superharmonic function for linear elliptic operator of second order, J. Math. Soc. Japan, 16 (1964), 299-306.

5. - Martin boundary for elliptic differential operators of second order in a manifold, J. Math. Soc. Japan, 16 (1964), 307-334.

6. M. Kawamura and M. Nakai, A test of Picard principle for rotation free densities, II, J. Math. Soc. Japan, 28 (1976), 323-342.

7. C. Miranda, Partial Differential Equations of Elliptic Type, Springer, 1970.

8. M. Nakai, Martin boundary over an isolated singularity of rotation free density, J. Math. Soc. Japan, 26 (1974), 483-507.

9. — A test of Picard principle for rotation free densities, J. Math. Soc. Japan, 27 (1975), 412-431.

10. - Picard principle and Riemann theorem, Tôhoku Math. J., 28 (1976), 277-292.

11. — Picard principle for finite densities, Nagoya Math. J., 70 (1978), 7-24.

12. M. Ozawa, Some classes of positive solutions of $\Delta u=P u$ on Riemann surfaces, I, Kōdai Math. Sem. Rep., 6 (1954), 121-126.

13. - Some classes of positive solutions of $\Delta u=P u$ on Riemann surfaces, II, Kōdai Math. Sem. Rep., 7 (1955), 15-20.

14. L. Sario and M. Nakai, Classification Theory of Riemann Surface, Springer, 1970.

Received March 3, 1978 and in revised form May 20, 1978.

Daido Institute of TeChNology

2-21 Daido, Minami, Nagoya 457

JAPAN 


\section{PACIFIC JOURNAL OF MATHEMATICS}

\section{EDITORS}

RICHARD ARENS (Managing Editor)

University of California

Los Angeles, California 90024

C. W. CURTIS

University of Oregon

Eugene, OR 97403

C. C. MOORE

University of California

Berkeley, CA 94720
J. DugundjI

Department of Mathematics University of Southern Californı Los Angeles, California 90007

R. Finn and J. Milgram Stanford University Stanford, California 94305

ASSOCIATE EDITORS

E. F. BECKENBACH

B. H. NeUmanN

F. WOLF

K. YoSHIDA

\section{SUPPORTING INSTITUTIONS}

UNIVERSITY OF BRITISH COLUMBIA CALIFORNIA INSTITUTE OF TECHNOLOGY

UNIVERSITY OF CALIFORNIA

MONTANA STATE UNIVERSITY

UNIVERSITY OF NEVADA, RENO

NEW MEXICO STATE UNIVERSITY

OREGON STATE UNIVERSITY

UNIVERSITY OF OREGON
UNIVERSITY OF SOUTHERN CALIFORNIA

STANFORD UNIVERSITY

UNIVERSITY OF HAWAII

UNIVERSITY OF TOKYO

UNIVERSITY OF UTAH

WASHINGTON STATE UNIVERSITY

UNIVERSITY OF WASHINGTON 


\section{Pacific Journal of Mathematics}

\section{Vol. 80, No. $1 \quad$ September, 1979}

Jeroen Bruijning and Jun-iti Nagata, A characterization of covering dimension by

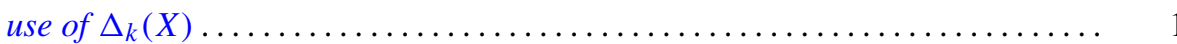

John J. Buoni and Albert Jonathan Klein, On the generalized Calkin algebra ...... 9

Thomas Ashland Chapman, Homotopy conditions which detect simple homotopy

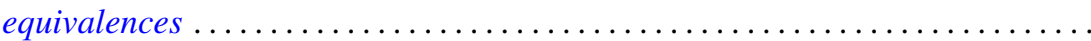

John Albert Chatfield, Solution for an integral equation with continuous interval

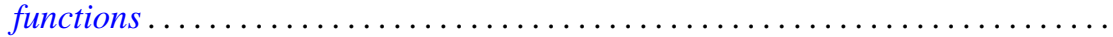

Ajit Kaur Chilana and Ajay Kumar, Spectral synthesis in Segal algebras on

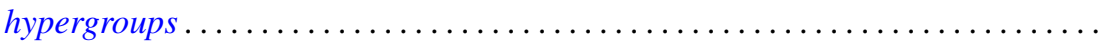

Lung O. Chung, Jiang Luh and Anthony N. Richoux, Derivations and

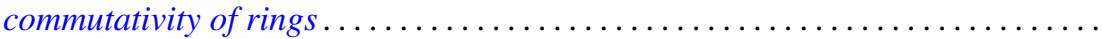

Michael George Cowling and Paul Rodway, Restrictions of certain function spaces to closed subgroups of locally compact groups .....................

David Dixon, The fundamental divisor of normal double points of surfaces........

Hans Georg Feichtinger, Colin C. Graham and Eric Howard Lakien,

Nonfactorization in commutative, weakly selfadjoint Banach algebras . . . . . . .

Michael Freedman, Cancelling 1-handles and some topological imbeddings ....... .

Frank E., III Gerth, The Iwasawa invariant $\mu$ for quadratic fields . . . . . . . . . . . . . .

Maurice Gilmore, Three-dimensional open books constructed from the identity

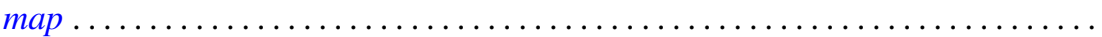

Stanley P. Gudder, A Radon-Nikodým theorem for $*$-algebras .

Peter Wamer Harley, III and George Frank McNulty, When is a point Borel? .

Charles Henry Heiberg, Fourier series with bounded convolution powers . .

Rebecca A. Herb, Characters of averaged discrete series on semisimple real Lie

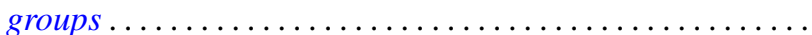

Hideo Imai, On singular indices of rotation free densities . .

Sushil Jajodia, On 2-dimensional CW-complexes with a single 2-cell . . .

Herbert Meyer Kamowitz, Compact operators of the form $u C_{\varphi}$

Matthew Liu and Billy E. Rhoades, Some properties of the Chebyshev method...

213

George Edgar Parker, Semigroups of continuous transformations and generating

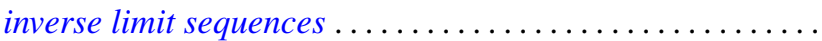

Samuel Murray Rankin, III, Oscillation results for a nonhomogeneous

equation ...

Martin Scharlemann, Transverse Whitehead triangulations ...

Gary Joseph Sherman, A lower bound for the number of conjugacy classes in a

finite nilpotent group

Richard Arthur Shoop, The Lebesgue constants for $\left(f, d_{n}\right)$-summability .

Stuart Jay Sidney, Functions which operate on the real part of a uniform

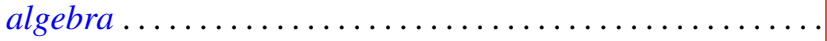

Tim Eden Traynor, The group-valued Lebesgue decomposition 